\title{
High ambipolar and balanced carrier mobility in regioregular poly(3-hexylthiophene)
}

\author{
S. A. Choulis, Y. Kim, J. Nelson, and D. D. C. Bradley ${ }^{\text {a) }}$ \\ The Blackett Laboratory, Imperial College London, London SW7 2AY, United Kingdom \\ M. Giles, M. Shkunov, and I. McCulloch \\ Merck NBSC Ltd, Chilworth Science Park, Southampton, United Kingdom
}

(Received 24 May 2004; accepted 11 August 2004)

\begin{abstract}
The carrier transport of carefully purified regioregular poly(3-hexylthiophene) films has been studied using time-of-flight photocurrent measurements. We find balanced ambipolar transport with a room-temperature mobility for holes of $3 \times 10^{-4} \mathrm{~cm}^{2} \mathrm{~V}^{-1} \mathrm{~s}^{-1}$ and for electrons of $1.5 \times 10^{-4} \mathrm{~cm}^{2} \mathrm{~V}^{-1} \mathrm{~s}^{-1}$ at electric fields $\geqslant 10^{5} \mathrm{~V} / \mathrm{cm}$. The transport is relatively field independent and weakly temperature dependent, pointing to a high degree of chemical regioregularity and purity. These factors make poly(3-hexylthiophene) attractive for use in a range of electronic applications. (0) 2004 American Institute of Physics. [DOI: 10.1063/1.1805175]
\end{abstract}

Conjugated polymers are of increasing interest as new materials for electronic applications, offering the potential for low fabrication cost, easy processing, and flexibility. Organic light-emitting diodes (OLEDs), field-effect transistors (FETs), photodiodes, and solar cells are all applications under intense study and first products are already emerging. One of the limitations for the utilization of such organic semiconductors is that they have relatively poor carrier transport properties in terms both of absolute mobility and with respect to the balance between hole and electron mobility. Low mobilities can limit practical applications, for instance, of OLEDs in passive matrix addressed displays due to low current densities in the space-charge-limited current regime, and unbalanced transport can reduce OLED-based display emission efficiency. Low mobility also impacts on switching speed in FETs and on solar cell efficiency, and again unbalanced transport has additional drawbacks in both of these applications.

In general, electron transport in conjugated polymers is much worse than hole transport, and tends to be highly dispersive, with no clear transit time observed in time-offlight (ToF) photocurrent measurements. ${ }^{1}$ Better electron transport polymers are, therefore, much needed in order to achieve the desired combination of enhanced and balanced carrier transport. We report here a study that reveals unexpected promise for poly(3-hexylthiophene) (P3HT) as a material with both good electron and hole transport. P3HT has been widely studied in the past and is well known as being an effective hole transport polymer, with higher hole mobility than many other conjugated polymers, including the poly(phenylenevinylene)s. ${ }^{2}$ Initially, however, rather low hole mobilities were reported for regiorandom P3HT. ${ }^{3}$ However, when head-to-tail regioregularly substituted P3HT became available, the hole mobilities were found to be dramatically improved. ${ }^{4-6}$ The increase in mobility was related to side-chain-induced self-organization into a well-ordered twodimensional lamellar structure. ${ }^{2}$ In contrast, electron transport has not been reported up to now in P3HT, most probably because electrons are heavily trapped. Molecular oxygen impurities have been proposed as one possible trap, ${ }^{7}$ but little

\footnotetext{
${ }^{\text {a)} E l e c t r o n i c ~ m a i l: ~ D . B r a d l e y @ i m p e r i a l . a c . u k ~}$
}

has been done to systematically address the issue. The extensive literature for P3HT highlights the importance of both structural and chemical influences on transport properties.

In this letter, we present results concerning carrier transport in regioregular (RR)-P3HT, obtained using ToF photocurrent measurements on single-layer device structures. The RR-P3HT used in this work was synthesized and carefully purified by Merck NBSC Ltd. The polymer number- and weight-average molecular weights were $2.8 \times 10^{4}$ and $4.4 \times 10^{4}$, respectively, and its regioregularity was measured to be greater than $96 \%$ by proton nuclear magnetic resonance. Importantly, the concentration of residual catalysts such as $\mathrm{Ni}$ and $\mathrm{Mg}$ was determined to be less than $10 \mu \mathrm{g} / \mathrm{g}$ after purification. Films of RR-P3HT were spin coated from chloroform solutions $(30 \mathrm{mg} / \mathrm{ml})$ onto indium tin oxide (ITO)-coated glass substrates. The ToF test structures were then completed by thermal evaporation of a shadow-masked, aluminum top contact, at a typical pressure of $10^{-6}$ mbar. ToF transients were measured at $20-70 \mathrm{~V}$ applied biases under pulsed (7 ns) illumination through the ITO electrode. Measurements were performed at $532 \mathrm{~nm}$ (where the absorption coefficient of RR-P3HT is $\alpha=2 \times 10^{5} \mathrm{~cm}^{-1}$ ) and $355 \mathrm{~nm}\left(\alpha=3 \times 10^{4} \mathrm{~cm}^{-1}\right)$ using the frequency-doubled and frequency-tripled outputs of a Nd:YAG laser (Quantel), respectively. No difference was observed in the electron mobility or its field/temperature dependence at these two excitation wavelengths. The resulting photocurrent was detected with a digital oscilloscope (Tektronix TDS 3052) and the carrier type under study was selected by changing the sign of the applied bias. The transit time $t_{\mathrm{tr}}$ for the arrival of carriers at the collecting electrode is related to the carrier mobility $\mu$ via the relation

$$
\mu=d /\left(E t_{\mathrm{tr}}\right),
$$

where $E$ is the applied electric field and $d$ the thickness of the RR-P3HT film. The samples were stored and measured in vасио. Measurements on films of different thickness (1.2, 1.4, 1.7, and $2.8 \mu \mathrm{m}$ ) showed that the observed mobilities were thickness independent. This demonstrates that the photocurrents are not range limited, but indeed reflect the drift of the carrier sheet across the entire sample. Consistent results 


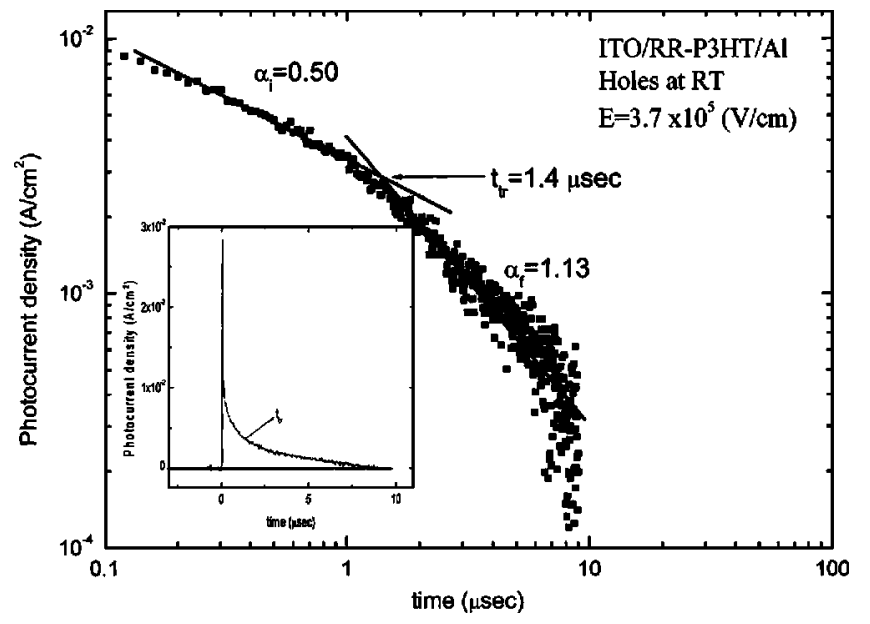

FIG. 1. A typical ToF hole transient for a RR-P3HT film. The data are plotted in both double-logarithmic (main plot) and double-linear (inset) representations. Measurements were performed at $295 \mathrm{~K}$ (RT) and for an applied electric field of $3.7 \times 10^{5}(\mathrm{~V} / \mathrm{cm})$. The slope values before $\left(\alpha_{i}\right)$ and after $\left(\alpha_{f}\right)$ the transit time $\left(t_{\mathrm{tr}}\right)$ are also reported in the plot.

were obtained for two different batches of RR-P3HT, synthesized at different times.

A typical hole current transient recorded at $E$ $=3.7 \times 10^{5} \mathrm{~V} / \mathrm{cm}$ and at room temperature $(\mathrm{RT})$ is shown in Fig. 1. The data are plotted in both double-logarithmic (main plot) and double-linear (inset) representations. The hole photocurrent transients are highly dispersive. They are characterized by temporal decays that show a "knee" only in the double-logarithmic plot. Nevertheless, the transit time $\left(t_{\mathrm{tr}}\right)$ can still be estimated from the intersection point at which the asymptotes to the log-log plot (pre- and post-knee) cross; this point is indicated by an arrow in Fig. 1. The mobility of the positive carriers that we deduce, namely $\mu_{h}$ $\approx 3 \times 10^{-4} \mathrm{~cm}^{2} / \mathrm{V} \mathrm{s}$ at $E \geqslant 10^{5} \mathrm{~V} / \mathrm{cm}$, is very similar to that found in previous ToF measurements on RR-P3HT. ${ }^{4-8}$

Figure 2 shows a corresponding typical RT electron current transient recorded with a reversed polarity field (to collect electrons) of amplitude $E=1.04 \times 10^{5} \mathrm{~V} / \mathrm{cm}$. The data are again plotted in both double-logarithmic (main plot) and double-linear (inset) representations. The electron photocur-

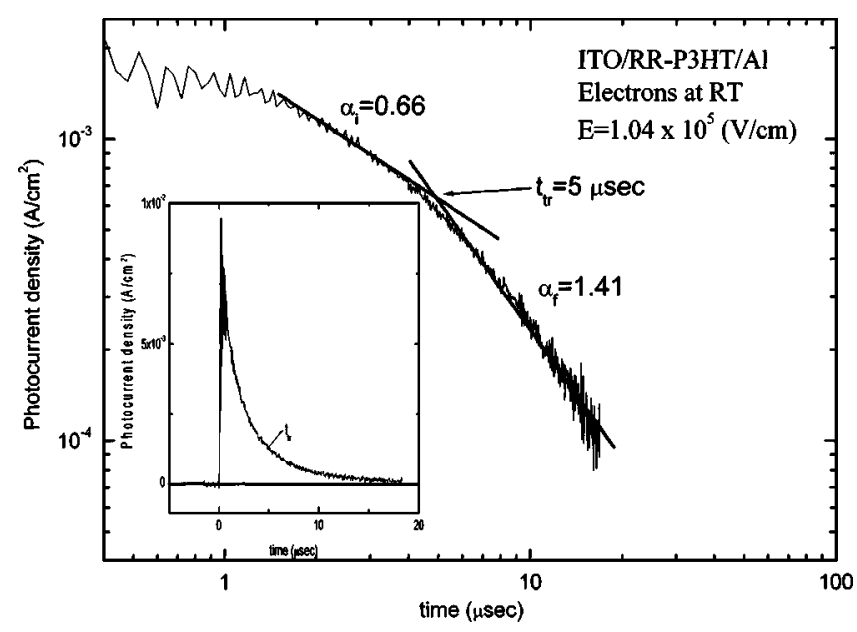

FIG. 2. A typical ToF electron transient for a RR-P3HT film, plotted in double-logarithmic and double-linear (inset) representations. Measurements were performed at $295 \mathrm{~K}$ at an applied electric field of $1.04 \times 10^{5}(\mathrm{~V} / \mathrm{cm})$. The slope values before $\left(\alpha_{i}\right)$ and after $\left(\alpha_{f}\right)$ the transit time $\left(t_{\mathrm{tr}}\right)$ are shown. Downloaded 03 Jun 2009 to 131.227.178.130. Redistribution subje

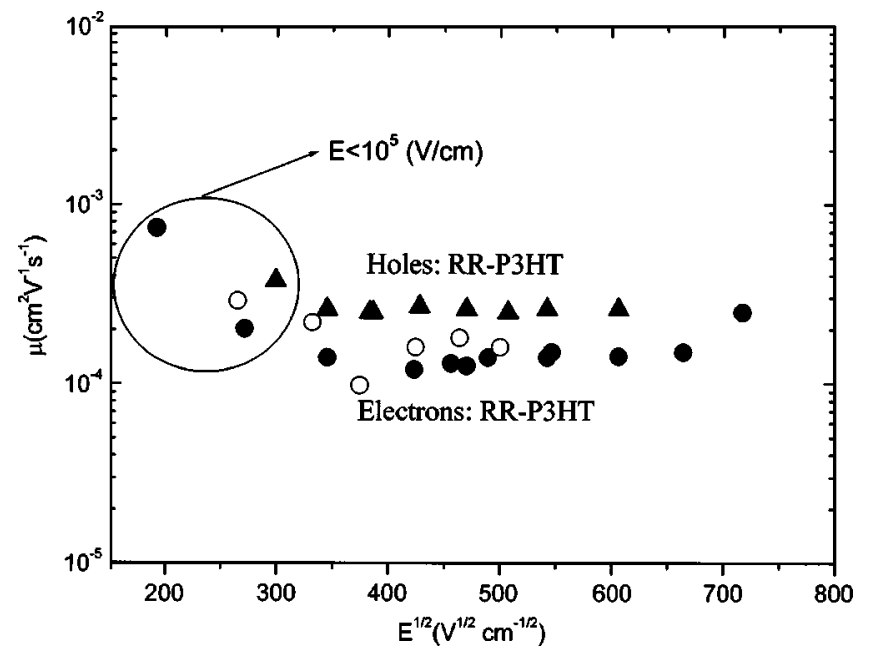

FIG. 3. Field dependence of the electron [exciting at $355 \mathrm{~nm}$ (filled-circles) and $532 \mathrm{~nm}$ (open-circles)] and hole [exciting at $355 \mathrm{~nm}$ (triangles)] mobilities in RR-P3HT at RT (295 K). Each data point shown was determined from the asymptotes to the corresponding double-logarithmic photocurrent transient plots.

rent transients are slightly less dispersive than those for holes, but again, the characteristic knee in the doublelogarithmic plot is needed to estimate $t_{\mathrm{tr}}$. As expected, logarithmic slopes for all the transients studied have values less than 1 and greater than 1 before and after $t_{\text {tr }}$, respectively. ${ }^{9}$ We also note that the collected charge was less than $6 \%$ of the sample capacitor charge in all cases, so that we could be confident that we are indeed working in a regime in which the electric field across the samples can be assumed constant [as required for Eq. (1)]. The electron mobility then deduced from Eq. (1) is of order $1.5 \times 10^{-4} \mathrm{~cm}^{2} / \mathrm{V} \mathrm{s}$ and is thus within a factor of approximately 2 of the hole mobility.

Figure 3 shows the field dependence of the RT electron and hole mobilities deduced from a sequence of ToF experiments performed with different bias fields. The mobility data are plotted as a function of the square root of the bias field (corrected for the built-in voltage), in accordance with the expectations of the Poole-Frenkel model. This square-root field dependence is often seen for molecular materials. ${ }^{10}$ Here, however, for $E \geqslant 10^{5} \mathrm{~V} / \mathrm{cm}$, we find a very weak field dependence for both electron and hole transport: The RRP3HT hole mobility is $\approx 3 \times 10^{-4} \mathrm{~cm}^{2} / \mathrm{V} \mathrm{s}$ and the electron mobility $\approx 1.5 \times 10^{-4} \mathrm{~cm}^{2} / \mathrm{V}$ s. For $E \leqslant 10^{5} \mathrm{~V} / \mathrm{cm}$ we note that both the electron and hole mobilities show a negative field dependence. Similar behavior is discussed in detail in Refs. 4, 5, and 8.

The influence of traps on charge transport depends on their energy relative to the transport states of the material. This is one of the reasons low-ionization-potential $\left(I_{p}\right)$ materials have found widespread use as organic hole transporters. ${ }^{11}$ For RR-P3HT the typical ionization potential reported in the literature, $I_{p} \approx 4.8 \mathrm{eV},{ }^{12}$ is quite low and good hole transport can be expected, as has already been reported. $^{4-7}$ The electron affinity $\left(E_{\alpha}\right)$ for RR-P3HT is $E_{\alpha}$ $\approx 2.9 \mathrm{eV}$. This low value for $E_{\alpha}$ means that the electron transport states are expected to lie close to typical impurity electron trap states that are often encountered in these materials. ${ }^{13}$ This suggests at first glance that RR-P3HT does not have the potential to be a good electron transport material, indeed electron transport has not been reported previ-
to AlP license or copyright; see http://apl.aip.org/apl/copyright.jsp 


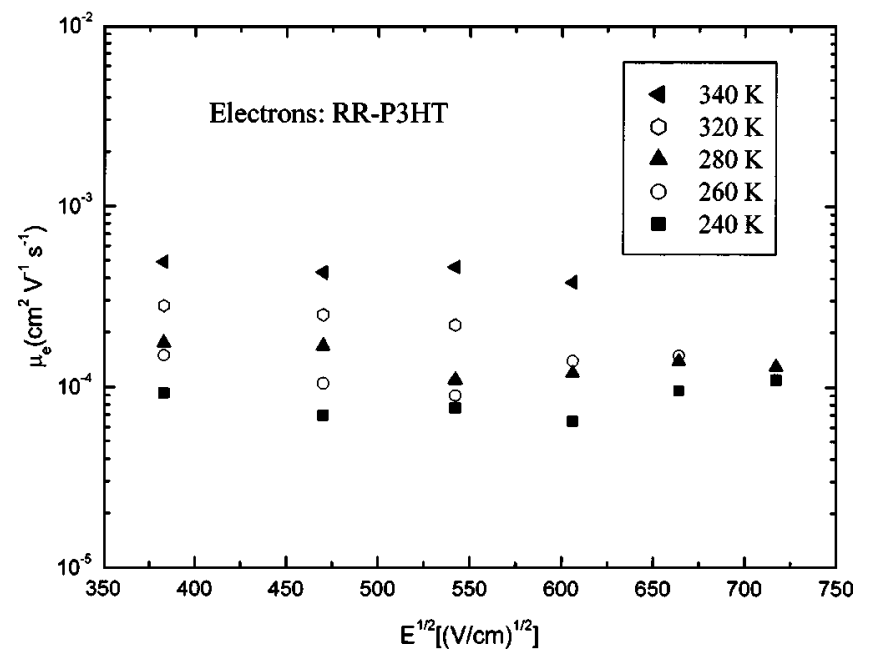

FIG. 4. Electron mobility in RR-P3HT as a function of temperature (240$340 \mathrm{~K})$. Each data point was determined from the asymptotes to the corresponding double-logarithmic photocurrent transient plots.

ously. However, from our measurements we have found that electron transport in RR-P3HT is comparable to the highest reported electron mobility values for other conjugated polymers, such as poly(9,9-dioctylfluoreneco-benzothiadiazole). ${ }^{1}$ This large and field-independent mobility indicates that our RR-P3HT has excellent purity and chemical/structural regularity. The high and balanced positive and negative charge carrier mobilities that we have observed for RR-P3HT have not been previously reported for any other conjugated polymer. Our observations are compatible with recent theoretical calculations, which indicate that similar charge transport characteristics are expected for electrons and holes in conjugated oligomers. ${ }^{14}$

In order to investigate microscopic mechanisms for the electron transport in RR-P3HT, we performed ToF photocurrent measurements for electrons as a function of temperature. Figure 4 shows the field dependence of the electron mobility in P3HT (plotted in Poole-Frenkel format) as a function of temperature. The electron mobility shows a relatively weak temperature dependence: It is found to increase from $\approx 9$ $\times 10^{-5} \mathrm{~cm}^{2} \mathrm{~V}^{-1} \mathrm{~s}^{-1}$ at $240 \mathrm{~K}$ to $\approx 5 \times 10^{-4} \mathrm{~cm}^{2} \mathrm{~V}^{-1} \mathrm{~s}^{-1}$ at $340 \mathrm{~K}$. The relatively weak temperature dependence is indicative of a low degree of energetic disorder for the transport active sites, consistent with a high degree of purity and chemical regularity. Nondispersive charge transport data in conjugated polymers has previously been analyzed with empirical models such as the Gaussian disorder model (GDM) and variants ${ }^{10}$ to yield values of empirical parameters believed to be indicative of the degree of disorder. In the present case, it has not been possible to analyze the data presented in Fig. 4 with the GDM with any confidence because of the small range of the mobility data (electron mobilities vary by less than one order of magnitude over a temperature range of $100 \mathrm{~K}$ ), and because of the relatively large uncertainty in the mobility values arising from the strongly dispersive transport that we measure. However the field and temperature dependence of the electron mobility in RRP3HT is reminiscent of other high mobility hole transporting polymers, suggesting a common mechanism. ${ }^{15}$

The relatively high electron and hole mobilities and balanced transport that we have observed in P3HT are expected to be important for a range of molecular electronic device applications. Although the photoluminescence quantum efficiency (QE) for P3HT is low, we anticipate that the comparable electron and hole mobilities will offer increased QE for RR-P3HT-based LEDs. High and balanced charge carrier mobilities are also an essential requirement for the construction of electrically pumped laser diodes ${ }^{11}$ and offer potential for fast switching FETs, both of which areas are of major interest for technological applications. In the latter context, we note that the absence of $n$-type transport in RR-P3HT FETs may in fact result from the high injection barrier for electrons and not from the intrinsic charge transport properties of the material. ${ }^{16}$ Furthermore, unbalanced mobilities in bulk hetero-junction solar cells may limit performance through space-charge buildup and recombination limitations on the short circuit current and fill factor. ${ }^{17}$ The high RT mobility presented here for holes in RR-P3HT suggests that the combination of RR-P3HT with fullerenes could offer more balanced transport within the blend and thus improve the polymer-fullerene device performance.

The experimental results presented provide a strong indication that ambipolar and balanced charge transport can be an intrinsic property of pure conjugated polymers. The RR-P3HT that we have studied exhibits good electron as well as hole transport with largely field-independent mobilities and only weak temperature dependence. The electron and hole mobilities obtained from RT ToF measurements $\left(E \geqslant 10^{5} \mathrm{~V} / \mathrm{cm}\right)$ were $\approx 1.5 \times 10^{-4} \mathrm{~cm}^{2} \mathrm{~V}^{-1} \mathrm{~s}^{-1}$ and $\approx 3 \times 10^{-4} \mathrm{~cm}^{2} \mathrm{~V}^{-1} \mathrm{~s}^{-1}$, respectively. Such well-balanced, ambipolar carrier transport offers the potential for RR-P3HT to find widespread use as the active organic semiconductor in a range of electronic applications.

The authors thank BP International for financial support.

${ }^{1}$ A. J. Campbell, D. D. C. Bradley, and H. Antoniadis, Appl. Phys. Lett. 79, 2133 (2001).

${ }^{2}$ R. Valaski, L. M. Moreira, L. Micaroni, and I. A. Hummelgen, J. Appl. Phys. 92, 2035 (2002).

${ }^{3}$ M. Kobashi and H. Takeuchi, Macromolecules 31, 7273 (1998).

${ }^{4}$ W. Takashima, S. S. Pandey, T. Endo, M. Rikukawa, and K. Kaneto, Curr. Appl. Phys.win 1, 90 (2001).

${ }^{5}$ G. Juska, K. Genevicius, K. Arlauskas, R. Osterbacka, and H. Stubb, Phys. Rev. B 65, 233208 (2002).

${ }^{6}$ H. Sirringhaus, P. J. Brown, R. H. Friend, M. M. Nielsen, K. Bechgaard, B. M. W. Langeveld-Boss, A. J. H. Spiering, R. A. J. Janssen, E. W. Meijer, P. Herwig, and D. M. de Leeuw, Nature (London) 401, 685 (1999).

${ }^{7}$ H. Andoniadis, M. A. Abkowitz, and B. R. Hsieh, Appl. Phys. Lett. 65, 2030 (1994).

${ }^{8}$ W. Takashima, S. S. Pandey, T. Endo, M. Rikukawa, N. Tanigati, Y. Yoshida, K. Yase, and K. Kaneto, Thin Solid Films 393, 334 (2001).

${ }^{9}$ H. Scher, M. F. Shlesinger, and J. T. Bendler, Phys. Today 44, 26, (1991).

${ }^{10}$ H. Bassler, Phys. Status Solidi B 175, 15, (1993).

${ }^{11}$ M. Redecker, D. D. C. Bradley, M. Inbasekaran, and E. P. Woo, Appl. Phys. Lett. 73, 1565 (1998).

${ }^{12}$ D. B. A Rep, A. F. Morpurgo, and T. M. Klapwijk, Org. Electron. 4, 201 (2003).

${ }^{13}$ A. J. Campbell, D. D. C. Bradley, and D. G. Lidzey, J. Appl. Phys. 82, 6326 (1997).

${ }^{14}$ J. L. Bredas, J. P. Calbert, D. A. Da. Silva Filho, and J. Cornil, Proc. Natl. Acad. Sci. U.S.A. 99, 5804 (2002).

${ }^{15}$ D. Poplavskyy, T. Kreouzis, A. Campbell, J. Nelson, and D. D. C. Bradley, Mater. Res. Soc. Symp. Proc. 725, 141 (2002).

${ }^{16}$ E. J. Meijer D. M. de Leeuw, S. Setayesh, E. van Veenendaal, B.-H. Huisman, P. W. M. Blom, J. C. Hummelen, U. Scherf, and T. M. Klapwijk, Nat. Mater. 2, 678 (2003).

${ }^{17}$ S. A. Choulis, J. Nelson, Y Kim, D. Poplavskyy, T. Kreouzis, J. R. Durrant, and D. D. C. Bradley, Appl. Phys. Lett. 83, 3812 (2003). 